\title{
Photon strength in spherical and deformed heavy nuclei
}

\author{
E. Grosse $e^{1,2}$, A. Junghans ${ }^{1}$, F. Bečvár $\check{r}^{3}$, E. Birgersson ${ }^{1}$, R. Massarczyk ${ }^{1}$, and G. Schramm ${ }^{1}$ \\ 1 Institute of Radiation Physics, Forschungszentrum Dresden-Rossendorf, 01314 Dresden, Germany \\ 2 Physics Department, Technische Universität, 01062 Dresden, Germany \\ 3 Faculty of Mathematics and Physics, Charles University, 18000 Prague 8, Czech Republic
}

\begin{abstract}
Information on the photon strength in heavy nuclei with mass $A>150$ will be given and compared to respective data. The photon strength function is a very important ingredient for statistical model calculations - especially when these are used to describe neutron capture. Several schemes for a transmutation of radioactive waste favor nuclear reactions with fast neutrons and these also influence the performance of various reactor types proposed to deliver nuclear energy together with only small quantities of such waste. Reactions with fast neutrons are less well studied as compared to those induced by thermal neutrons. As they are not easily accessible experimentally, reference is often made to calculations using the statistical model. Photon emission probabilities are needed as input to such calculations aiming for predictions on fission to capture ratios. Results of approximate calculations for radiative neutron capture will be presented.
\end{abstract}

\section{Introduction}

Nuclear properties near the neutron separation energy $S_{\mathrm{n}}$ - below and above - have attracted much attention not only as supplement to nuclear structure studies at low energy but especially due to their importance in nuclear astrophysics as well as nuclear technology: (i) In the cosmic synthesis of the elements the interplay of radiative neutron capture and thermally induced neutron emission plays an important role. (ii) In advanced systems for the generation of nuclear energy and in equipment for the transmutation of nuclear waste fast neutrons, emitted during fission, induce reactions in the fuel as well as in structural materials. To solve relevant problems, eventually emerging in both cases, the respective cross sections have to be known, but their direct measurement is often rather difficult or even impossible. It is not primarily fundamental theory what is needed here, but rather theory based systematic parameterizations for the extrapolation and generalization of data obtained in nuclei more easily available for experimental study.

The photon strength [1] just above and below the particle separation energies is a crucial quantity here. In contrast to the rather extensive experimental studies of the photon strength in the isovector giant dipole resonance (GDR) region well above the particle thresholds [2], similarly detailed observations for the excitation region below them are still surprisingly scarce and various proposals have been made for their parameterization [3]. When used to describe the photon strength below particle thresholds a two-resonance fit to GDR data gives satisfactory results in well deformed nuclei [4], whereas single Lorentzians do not to always agree to low-energy data [5] in less deformed ones.

The work described here is motivated by the observation [6] that the extraction of electric dipole strength as often applied in the analysis of data for heavy nuclei is based on a fit of one Lorentz curve to the peak region of the GDR [2]. Using theoretical arguments [7], the resulting strength is then reduced at lower excitation energies. It then apparently agrees to some primary photon data from neutron capture [6] assumed to deliver photon strength information at such low energy. This approach

This is an Open Access article distributed under the terms of the Creative Commons Attribution-Noncommercial License 3.0, which permits unrestricted use, distribution, and reproduction in any noncommercial medium, provided the original work is properly cited. 
was reassessed to derive a description of the photon strength observed by inelastic photon scattering [8]. Thus the data in the GDR region were reanalyzed [5], the 'old' fits were ignored and instead a parameterization was achieved in agreement to the dipole sum rule (DSR) [9]. Here previous experimental data [10] had to be renormalized slightly to adjust them to newer measurements [11]. To avoid any sensitivity on particle parameters only energies were regarded at which transmission coefficients are unity and data from below threshold were taken either from photon scattering or from average resonance neutron capture (ARC). To test this 'new' parameterization at low photon energy we now also calculate its effect on radiative capture cross sections and we will in the following compare the resulting predictions to respective data.

\section{The photon strength function and radiative capture}

The photon strength function (PSF) $f_{\lambda}$ is related to the average cross section for photon absorption from 0 to anenergy $E_{x}$

$$
\left\langle\sigma_{\gamma}\right\rangle=f_{\lambda}\left(E_{x}\right)(2 \lambda+1)(\pi \hbar c)^{2} E_{\gamma}^{2 \lambda-1} .
$$

The Axel-Brink hypothesis [1] states that the strength only depends on the transition energy $E_{\gamma}$ and that the same $f_{\lambda}$ holds for absorption and emission (following e.g. radiative capture) such that for multipolarity $\lambda$ an average for the decay width $\Gamma_{\lambda}$ from $E_{i}$ to $E_{f}=E_{i}-E_{\gamma}$ can be calculated from $f_{\lambda}$ by setting:

$$
\left\langle\Gamma_{\lambda}\right\rangle=f_{\lambda}\left(E_{\gamma}\right) D E_{\gamma}^{2 \lambda+1}, \quad D=1 / \rho\left(E_{i}\right) .
$$

As was shown previously [5], a parameterization of the dipole strength in the GDR can account for deformation effects in a direct way by using independent information from spectroscopic studies on the deformation parameters $\beta$ and $\gamma$. As the GDR dipole oscillation is fast as compared to vibrational or rotational modes the widening due to the deformation can be treated adiabatically. This can be done by inserting [12] deformation parameters derived from B(E2) values in the Hill-Wheeler expression [13] for the three axes, which are inversely proportional to the three oscillation frequencies. Different approaches exist, but they do not disagree much in their prediction of the GDR shape. One alternative is the method proposed by Kumar [14] for the analysis of Coulomb excitation data [15], which has the advantage of delivering information about deviations from axial symmetry. We also make use of the fact [16], that the parameters for quadrupole deformation and triaxiality correlate surprisingly well in the sense, that small deformation favors large triaxiality. A very convincing aspect of including triaxiality here is the fact, that the resulting GDR widths, which characterize the spreading, are depending only very smoothly on the nuclear charge and mass number. This has the important consequence that the extrapolations of the dipole strength to low energy are very similar for well deformed and spherical nuclei in their neighborhood as well as for the intermediate nuclei with small $\beta$, which are often considered soft against triaxial shape changes.

Distributing the integrated dipole strength $I_{D S R}$ as predicted by the dipole sum rule (DSR) evenly over the three components we obtain the expression:

$$
f_{1}\left(E_{\gamma}\right)=\sum_{k=1,3} \frac{2 \cdot I_{k}}{3 \pi(\pi \hbar c)^{2}} \frac{E_{\gamma} \cdot \Gamma_{k}}{\left(E_{k}^{2}-E_{\gamma}^{2}\right)^{2}+E_{\gamma}^{2} \Gamma_{k}^{2}}, \quad I_{1}=I_{2}=I_{3}=\frac{1}{3} I_{D S R}
$$

which we compare to various data to determine their deviation from the sum rule and to find out to what extent it describes the dipole strength at low energies. The resonance integral $I=\int \sigma_{\gamma} \cdot d E_{\gamma}$ is the appropriate measure of the size of the resonant cross section. The GDR centroid energies $E_{0}$ of a spherical nucleus with $\mathrm{A}$ and $\mathrm{Z}$ are well predicted by the FRDM [17] with the symmetry-energy constant $\mathrm{J}=32.7 \mathrm{MeV}$ and the surface stiffness $\mathrm{Q}=32.7 \mathrm{MeV}$ taken from the fit to masses [18]. The additional parameter $\mathrm{m}_{\mathrm{eff}} \cdot \mathrm{c}^{2}=874 \mathrm{MeV}$ gives the best fit [5] to all nuclei with $\mathrm{A}>80$. Generalizing a suggestion of Bush and Alhassid [19], originally formulated for the three components of the GDR in one deformed nucleus belonging to the three orthogonal nuclear axes, we parameterize the spreading width for all nuclei with $\mathrm{A}>80$ by $\Gamma_{k}\left(E_{k}\right)=0.05 \cdot E_{k}^{1.6}$ with the exponent 1.6 derived from hydrodynamical considerations [19] and the proportionality factor obtained from a fit [5] to more than 
20 different nuclei with $A>80$. Thus, the spreading width depends only on the centroid energies $E_{k}$ along the three major axes and thus smoothly on $\mathrm{A}$ and $\mathrm{Z}$.

A further test of the predicted strength function is obtained from radiative capture data for neutron energies from the region of unresolved resonances $R$, which are low enough that no other channels are open and thus the proper selection of an optical potential does not play a role. Combining the semi-classical expression for the angular momentum $\ell h=r p_{n}$ to the approximation for replacing $\left\langle\Gamma_{n} \Gamma_{\gamma} / \Gamma_{t o t}\right\rangle_{R}$ by $\left\langle\Gamma_{\gamma}\right\rangle_{R}$, the average capture cross section can be approximated by $[1,20]$ :

$$
\langle\sigma(n, \gamma)\rangle \approx 2 \pi^{2}(\lambda+r)^{2} \sum_{R} \rho\left(E_{R}\right)\left\langle\Gamma_{\gamma}\right\rangle_{R} \approx 2 \pi^{2}(\lambda+r)^{2} \rho\left(S_{n}\right) \int_{0}^{E_{R}^{\max }} e^{E_{n}-E_{\gamma} / T} E_{\gamma}^{3} f_{1}\left(E_{\gamma}\right) d E .
$$

Here we average over many resonances $R$ and sum over all final states $f$ reached by the primary capture $\gamma$-rays. We also apply the constant temperature model [21] for the level density $\rho$ with its advantage of $\mathrm{T}$ being a straightforward measure for its energy dependence. As the approximation (4) is dominated by $1 / \mathrm{T}, f_{1}\left(E_{\gamma}\right)$ and $\rho\left(S_{n}\right)$, a comparison to respective data allows conclusions on lower limits for these quantities. Taking $\rho\left(S_{n}\right)$ directly from neutron capture resonance data [20] assumes the spin distribution at $S_{n}$ to remain valid within the energy range as considered here.

\section{Comparison to experimental data}

To cover a wide range in A and in deformation we present here results for ${ }^{156} \mathrm{Gd},{ }^{181} \mathrm{Ta},{ }^{197} \mathrm{Au},{ }^{206} \mathrm{~Pb}$ and ${ }^{238} \mathrm{U}$. For these nuclei strength function data in the GDR region [10,22] and below the neutron threshold are available. If for the latter ARC-data are used, their cross section had to be normalized such that strength below the gamma ray detection limit is accounted for [23,24]. In the photon scattering studies at ELBE [8] both the resolved strength and unresolved strength were separated by a deconvolution procedure. The radiative capture data also used in the following to test the 'new' photon strength parameterization (3) are taken from the literature; in the comparison to eq. 4 the level density at $S_{n}$ [20] and its slope T [21] enter directly. The integrand peaks at photon energies of 3-5 MeV such that one is especially sensitive to contributions to the strength in this energy range.

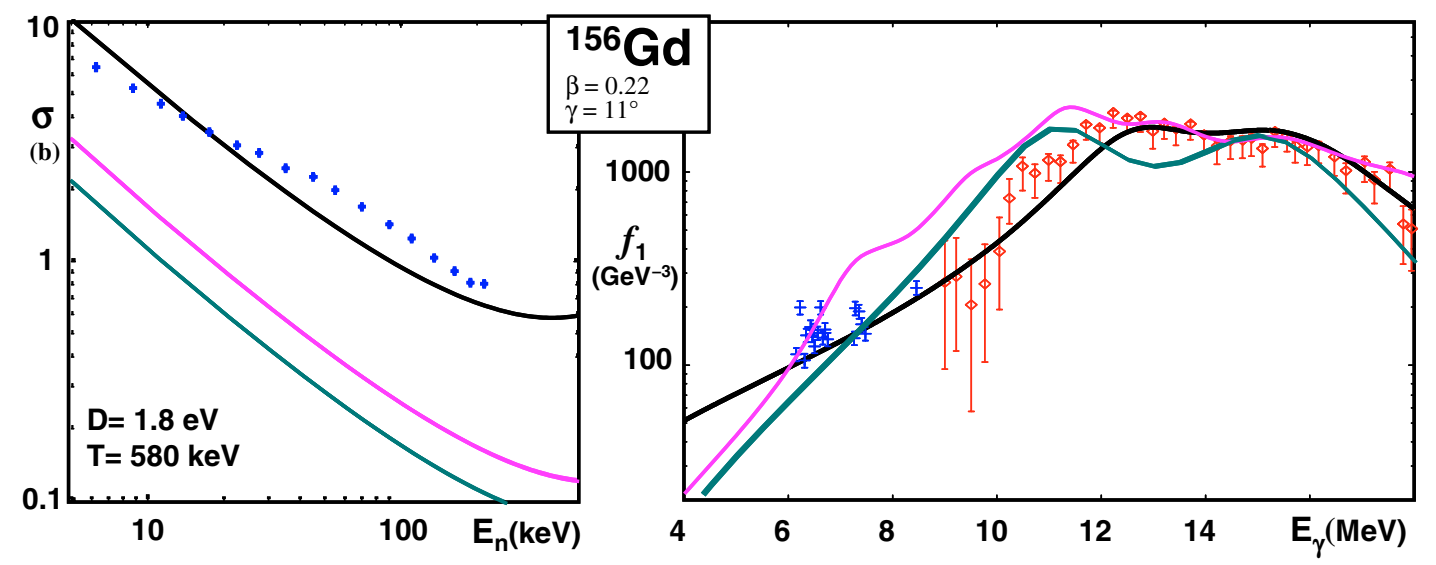

Fig. 1. The right panel shows the dipole strength below and above the neutron threshold for ${ }^{156} \mathrm{Gd}$. The experimental data from ARC $[+, 24]$ and $(\gamma, \mathrm{n})[\diamond, 25]$ are averaged to reduce the influence of Porter-Thomas fluctuations. The black line represents our parameterization whereas the magenta line corresponds to E1-strength functions as determined within the QRPA model based on the SLy4 Skyrme force as presented in RIPL-2 [3]. The analytic expression 'EGLO' as tabulated in RIPL-2 [3] is depicted in green. In the left panel the corresponding predictions for radiative capture by ${ }^{155} \mathrm{Gd}$ are shown combined to respective data [26]. 

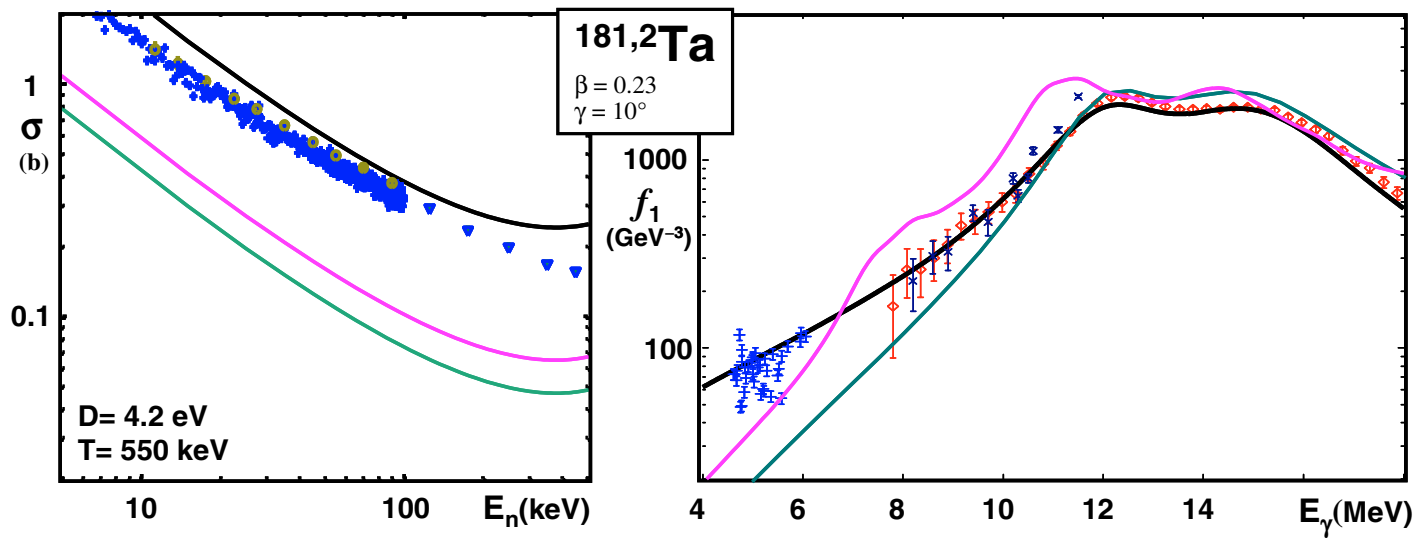

Fig. 2. Radiative capture by and dipole strength in ${ }^{181}$ Ta: Data for above the neutron threshold $[\diamond, 10 ; \mathbf{x}, 28]$ are combined to experimental data from ARC $[\mathbf{v}, 26 ;+, 24]$. The lines have the same meaning as in Fig. 1.

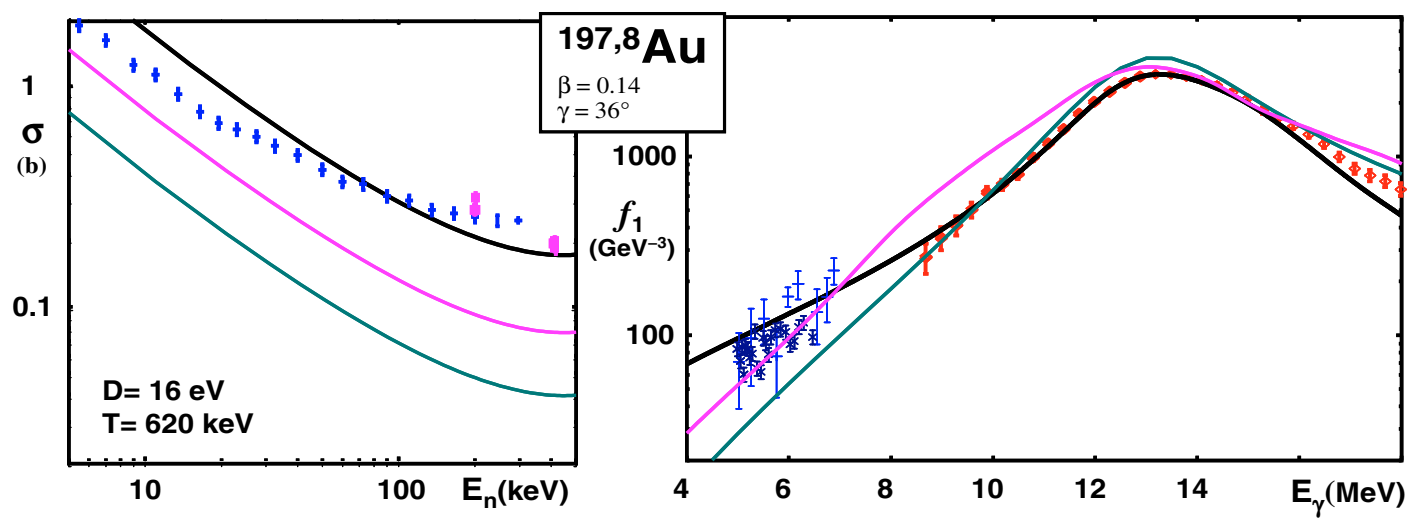

Fig. 3. Radiative capture by and dipole strength below and above the neutron threshold in ${ }^{197} \mathrm{Au}$ (or ${ }^{198} \mathrm{Au}$ resp.). The experimental data from ARC $[+, 10 ; *, 29 ;+, 30],(\gamma, \mathrm{n})[\diamond, 10]$ and photon scattering $[\diamond, 1]$ are averaged over $0.2 \mathrm{MeV}$. They agree to each other within their error bars. The lines have the same meaning as in Fig. 1.
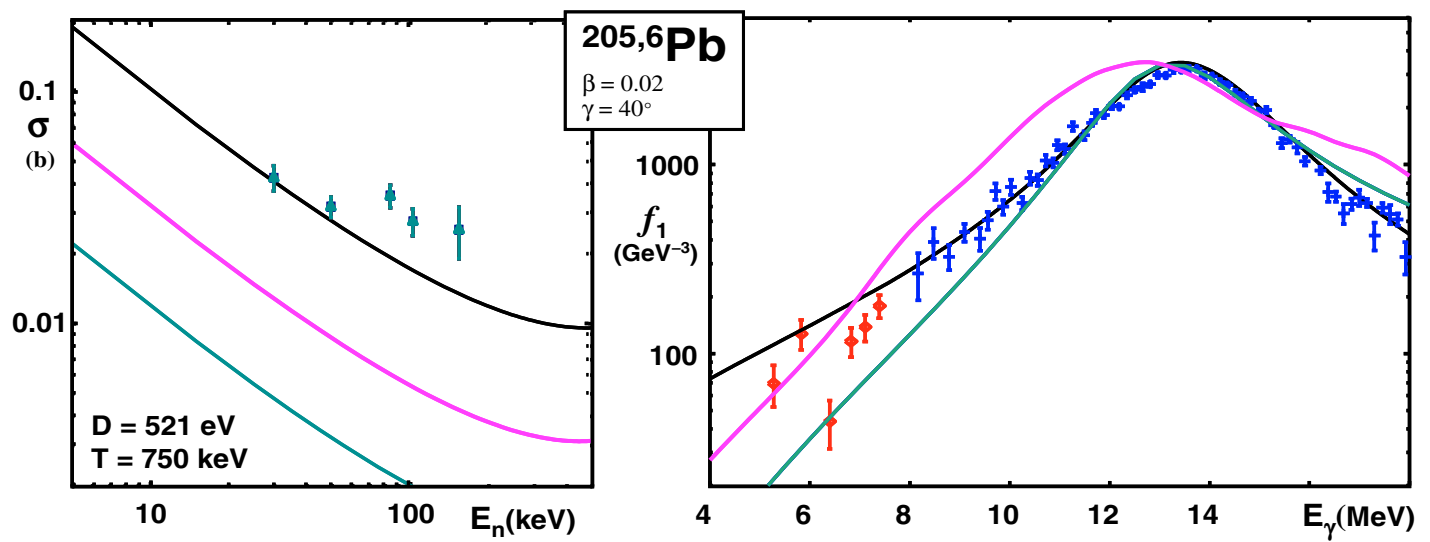

Fig. 4. Dipole strength in ${ }^{206} \mathrm{~Pb}$ as compared to photon absorption data for above the neutron threshold $[+, 31]$ are combined to experimental data from photon scattering [ $\downarrow, 32]$. The left panel depicts the radiative neutron capture of ${ }^{204} \mathrm{~Pb}$ [33] with the lines having the same meaning as in Fig. 1. 


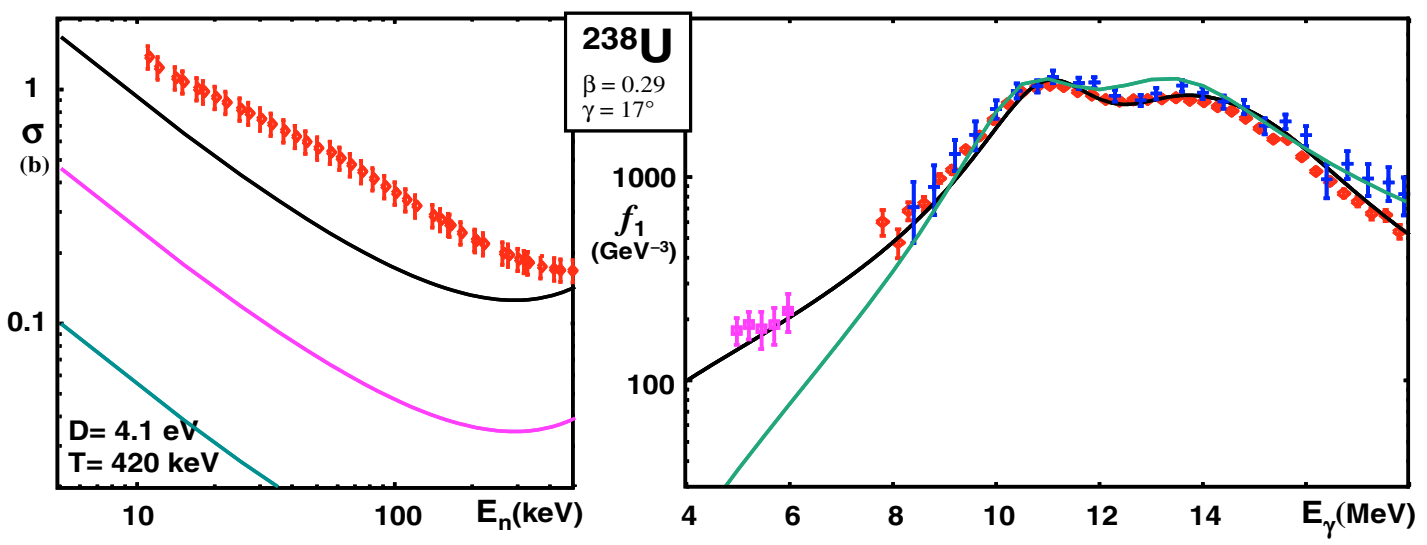

Fig. 5. Radiative capture by ${ }^{237} \mathrm{U}$ (left, extracted from (n,2n) data [[34]]) and dipole strength in ${ }^{238} \mathrm{U}$ (right): Data for above the neutron threshold $[\diamond, 10 ;+, 25]$ are combined to data from photon scattering $[\bullet, 35]$. The black and cyan lines have the same meaning as in Fig. 1 and the magenta line corresponds to MLO1 in RIPL-2 [3].

Obviously the two photon strength functions taken from RIPL-2 [3] disagree to photon absorption data and significantly underpredict the neutron capture cross sections. The other of the PSFs proposed there do not much better and the Hauser-Feshbach type calculations used e.g. in ref. [27] may have deficiencies as well due to the fact that they also rely on the 'old' analysis [2] of ( $\gamma, n)$ data.

\section{Conclusions}

From the favorable comparison of our parameterization to the experimental data for photon induced as well radiative capture processes in nuclei with various shapes and level densities we conclude:

The giant dipole resonance has very much the same properties in all heavy nuclei when their deformation is properly accounted for and its spreading width varies only smoothly with the resonance energies $E_{k}$ and not with the photon energy $E_{\gamma}$. Here our ansatz is at variance e.g. to calculations performed in a RPA scheme [3,36] and on the EGLO-prescription [3,37] based on theoretical arguments [7] derived from the theory of Landau liquids; both result in a rather steep decrease of the strength at lower energy. In contrast to previous work [37] our parameterization describes the dipole strength in near magic and in well deformed nuclei with the same formalism. For transitional and triaxial nuclei it also works well, if spectroscopic information on their deformation is properly included. The strength in the observed range deviates by less than $10 \%$ from the sum rule [9] and extra 'pygmy' fragments below the GDR contribute a few percent only.

As indicated for ${ }^{197} \mathrm{Au}$ the strength data below $\mathrm{S}_{n}$ as obtained from photon scattering [1] and from gamma emission after ARC [10] agree to each other - in accordance to the Axel-Brink hypothesis. From the radiative neutron capture results presented here one finds further support for it and one also learns that our parameterization is at least a good approximation also at photon energies below $4 \mathrm{MeV}$ dominating this process. From photon scattering one knows that at these low energies various discrete levels can be excited by E1, E2 or M1 photons and more stringent conclusions should especially include magnetic dipole excitations, which have been observed to appear in distinct and narrow energy domains. Respective data have been presented recently [38] in combination to calculations for spin-flip and orbital modes; the latter ones strongly depend on nuclear deformation. Apparently the M1 strength cannot be described by a single Lorentzian and first estimates indicate an increase of capture cross sections especially for deformed nuclei, but there clearly is a need for more detailed investigations.

Concerning the use of photon strength parameterizations in Hauser-Feshbach calculations for nuclear astrophysics or for nuclear energy applications the parameterization as presented here seems to be superior to the various prescriptions proposed previously e.g. by RIPL-2 [3]. As these strength functions - as well as variations given in the codes TALYS [39] and NON-SMOKER [27] - are based on the 'traditional' extraction of GDR parameters [2], they should be replaced (or used with special care), as we have pointed out the shortcomings of this extraction method. 


\section{References}

1. P. Axel, Phys. Rev. 126, 671 (1962); G.A. Bartholomew et al., Adv. Nucl. Phys. 7, 229 (1972).

2. S. Dietrich and B.L. Berman, At. Data Nucl. Data Tables 38, 199 (1988).

3. T. Belgya et al., IAEA-1506 (2006), www-nds.iaea.org/ripl-2/.

4. S. Raman et al., Phys. Rev. C 23, 2794 (1981); F. Bečvář et al., Phys. Rev. C 52, 1278 (1995).

5. A.R. Junghans et al., Phys. Lett. B 670, 200 (2008).

6. J. Kopecky and M. Uhl, Phys. Rev. C 41, 1941 (1990); J. Kopecky et al., Phys. Rev. C 47, 312 (1993).

7. S.G. Kadmenskii, V.P. Markushev and V.I. Furman, Sov. J. Nucl. Phys. (Yad. Fiz.) 37 (1983) 165.

8. G. Rusev et al., Eur. Phys. J. A 27, 171 (2006); R. Schwengner et al., Phys. Rev. C 76, 034321 (2007); G. Rusev et al., Phys. Rev. C 77, 064321 (2008); A. Wagner et al., Jour. of Phys. G 35, 014035 (2008).

9. J. Levinger and H.A. Bethe, Phys. Rev. 78, 115 (1950).

10. R. Bergere et al., Nucl. Phys. A 121, 463 (1968); R. Bergère et al., Nucl. Phys. A 133, 417 (1969); A. Veyssière et al., Nucl. Phys. A 159, 561 (1970); A. Leprètre et al., Nucl. Phys. A 175, 609 (1971); A. Veyssière et al., Nucl. Phys. A 199, 45 (1973); P. Carlos et al., Nucl. Phys. A 219, 61 (1974).

11. B.L. Berman et al., Phys. Rev. C 36, 1286 (1987); C. Nair et al., Phys. Rev. C 78, 055802 (2008); M. Erhard et al., Phys. Rev. C 81, 034319 (2010).

12. S. Raman et al., At. Data Nucl. Data Tabl. 78, 1 (2001).

13. D.L. Hill and J.A. Wheeler, Phys. Rev. 89, 1102 (52).

14. K. Kumar, Phys. Rev. Lett. 28, 249 (1972).

15. M. Zielinska et al., Nucl. Phys. A 712 , 3 (2002).

16. W. Andrejtscheff and P. Petkov Phys. Rev. C 48, 2531 (1993).

17. W.D. Myers et al., Phys. Rev. C 15, 2032 (1977).

18. P. Möller et al., At. Data Nucl. Data Tables 59, 185 (1995).

19. B. Bush and Y. Alhassid, Nucl. Phys. A 531, 27 (1991).

20. H. Feshbach, D.C. Peaslee and V.F. Weisskopf, Phys. Rev. 71, 145 (1947); D.J. Hughes et al., Phys. Rev. 91, 1423 (1953) J.E. Lynn, Neutron Resonance Reactions; CLARENDON PRESS, Oxford (1968); S.F. Mughabghab, Atlas of Neutron Resonances; ELSEVIER, Amsterdam (2006).

21. T. von Egidy and D. Bucurescu, Phys. Rev. C 80, 054310 (2009).

22. EXFOR database: http://cdfe.sinp.msu.ru/exfor/index.php.

23. R. Casten et al., Nucl. Phys. A 316, 61 (1979).

24. S.F. Mughabghab, C.L. Dunford, Phys. Lett. B 487, 155 (2000).

25. G.M. Gurevich et al., Nucl. Phys. A 273, 326 (1976); G.M. Gurevich et al., Nucl. Phys. A 338, 97 (1980).

26. K.Wisshak et al., Phys. Rev. C 52, 2762 (1995); K.Wisshak et al., Phys. Rev. C 69, 055801 (2004).

27. T. Rauscher and F.-K. Thielemann, At. Data Nucl. Data Tabl. 88 (2004) 1.

28. S. Goko et al, Phys. Rev. Lett. 96, 192501 (2006).

29. A.N. Davletshin et al., Atom. Eng. 65, 343 (1988).

30. N. Yamamuro et al., Nucl. Science and Tech. 20,797 (1983).

31. R.R. Harvey et al., Phys. Rev. 136, B126 (1964).

32. R.M. Laszewski and P. Axel Phys. Rev. C 19, 342 (1979).

33. R.L. Macklin and J. H. Gibbons, Phys. Rev. 159, 1007 (1967).

34. M. Caner, Nucl. Sci. Eng. 59, 395 (1976).

35. Y. Birenbaum et al, Phys. Rev. C 36, 1293 (1987).

36. S. Goriely, E. Khan, Nucl. Phys. A 706, 217 (2002); E. Khan et al., Nucl. Phys. A 694, 103 (2001).

37. J. Kopecky and M. Uhl, Phys. Rev. C 41, 1941 (1990); J. Kopecky et al., Proc. of a Meeting on (n, $\gamma)$, Bologna, Italy ECN-RX-94-103 (1994).

38. K. Heyde et al., arXiv 1004-3429 (2010).

39. A.J. Koning, S. Hilaire and M.C. Duijvestijn, TALYS-1.0., Nuclear Data for Science and Technology, eds O. Bersillon et al., (EDP Sciences, Nice, 211, 2008). 